\title{
Tladi wa Dikgati: Sengwalo sa phatose ('pathos')
}

\section{T.S. Kekana}

\section{M.J. Mojalefa*}

Department of African Languages, University of Pretoria, Pretoria, 0002, Email ${ }^{*}$ Corresponding author e-mail address: jerry.mojalefa@up.ac.za thupana.kekana@gmail.com

\section{Kakaretšo}

Ka lebaka la gore ditiragalo tša phatose di fela di hlakahlakanywa bobading le bosekaseking bja masetlapelo, taodišwane ye e yo ahlaahla kanegelo ya phatose ge e nepiša mohuta wa sengwalo sa kwešišano sa go lebana le phatose. Bjale ditiragalo tša phatose di tlo hlalošwa ge di lebane le kwelobohloko le manyami a magolo a go nyalana le lehu la molwantšhwa yoo karolo ya setšhaba le mmadi ba ikgwarantšego le yena. Moanegwa yoo o welwa ke matshwenyego a magolo ka kudu go feta moanegwagolo wa masetlapelo.

TIhalošo ya ditiragalo tša sengwalo sa phatose, e yo kgonthišwa ka go sekaseka kanegelo ya go ngwalwa ke Phatudi ka ngwaga wa 1964 gore go tle go gatelelwe tharollo ya kgakanego le tlabego tšeo di felago di rotoša hlogo ya go lebana le go ba gona ga kwelobohloko dingwalong tša mohuta wo wa masetlapelo le phatose. Nyakišišo e yo kgonthiša ge thokofalo ya Tladi (moanegwathwadi wa phatose) e tsoša tlabego le kgakanego ka tsela ya kwelobohloko ye kgolo go setšhaba.

\section{Abstract}

Narratives of pathos are often confused with tragic narratives. This article analyses a novel of pathos, Phatudi's novella Tladi wa Dikgati (1964) as a moral narrative. Pathos is explained in terms of how it evokes pity for the protagonist's pain, which culminates in the death of the main character, 
who is a loyal and much appreciated member of society and has captured the esteem of the readers.

The main character in a narrative of pathos experiences serious problems which differ from those experienced by the main characters of tragic narratives. A detailed explanation of pathos is given in an analysis of Phatudi's (1964) novella in order to solve the confusion that emanates from the pity shown by readers of the two types of narratives (tragic narratives and narratives of pathos). The discussion focuses on the death of the protagonists of pathos.

\section{Matseno}

Masetlapelo ke mohuta wa dikanegelo tša prosa. Kgopolo ye e thekgwa ke Steiner (1961: 16) ge a re sereto goba prosa se ka bitšwa masetlapelo ka lebaka la morero wa sona. Go tšwela pele go lemogwa gore masetlapelo le Aristotle ke monwana le lenala, ebile ke ntepa le lešago. Se se hlolwa ke gore Aristotle e le yena yo a thomilego go ngwala ka ga masetlapelo. Ka nako ya boAristotle masetlapelo a be a nepiša terama fao molwantšhwa a bego a feleletša ka lehu. Ge a hlaloša seo masetlapelo e lego sona Aristotle (Leech, 1969: 1) o re:

Tragedy is an imitation of an action that is serious and has magnitude, which is complete in itself, in language with pleasurable accessories, each kind brought in separately in the parts of the work, in a dramatic not in a narrative form, involving incidents arousing pity and fear, wherewith to accomplish its catharsis of such emotions.

Fa Aristotle o gatelela ntlha ya go re masetlapelo a hlohleletša letšhogo le kwelobohloko. Godimo ga moo masetlapelo ao a bolelwago fa ke Aristotle a be a lekana ka maatla nakong yeo. Ka go realo ge, ge go hlokomedišišwa gabotse masetlapelo a, go lemogwa gore therešo ke gore ga a lekane ka maatla. Go na le masetlapelo ao a bago gona ka ge go hlagile lehu. Masetlapelo a mohuta wo ke ao a hlalošwago fa ke Aristotle. Go feta fao go na le ao a tšweletšago kwelobohloko ya go feta le lehu. Ge a thekga ntlha yeo Jafta (1996: 8) o re: 
Tragedy is associated with a particular form in literature which has come to be accepted as drama or a play while the tragic extends beyond the confines of drama. The tragic is used to mean a type of experience, a sense, a vision of some kind.

Rateori yo o tiišetša kgopolo ya go re le ge Aristotle e le pulamadibogo dingwalong tša masetlapelo go lemogilwe gore masetlapelo ga a lekane; a mangwe a na le maatla go feta a mangwe. Taba ye e hlolela babadi kgakanego ye kgolo. Ge go le bjalo nyakišišo ye, e tlo leka go rarolla bothata bjo bja go tšweletšwa ke Aristotle bja go re masetlapelo ka moka ga ona a lekana ka maatla. Go realo go bolela gore go na le mehuta ye mebedi ya masetlapelo, e lego (a) masetlapelo ao a felelago ka lehu, e lego se ka Seisimane se bitšwago 'tragedy' le (b) masetlapelo a go lebana le kwelobohloko ya go feta le lehu, seo Jafta (1996) a se bitšago masetlapelo a maatla, kgopolo yeo Maisimane a e bitšago 'pathos'. Le ge go le phapano ye ya thalošo ya masetlapelo, nyakišišo e yo nepiša karolwana ya (b), e sego go fapantšha dikarolwana tšeo tše pedi tša masetlapelo ka ge nepo ya taodišwana ye e sa lebana le papetšo ya masetlapelo. Taba yeo le yona e tlo thuša go tla ka tharollo ye e kgotsofatšago ya bothata bjo bja Aristotle. Tharollo ye bjalo e tla thongwa ka go fo nepišwa pele le thalošo ya kgopolo ya phatose.

\section{Phatose}

Taodišwana ye e yo tsinkela sengwalo sa phatose ge se lebane le mohuta wa kanegelo ya sengwalo sa kwešišano (morality). Kwešišano e lebane le mokgwa wa go anega sengwalo ka tsela ya go ruta melao ya bophelo (boitshwaro) (Groenewald, 1993: 37). Ka fao ge go tsinkelwa mekgwa ye mebedi ya go anega dingwalo, go bolelwa gape le ka sengwalo sa phatose. Le ge go le bjalo go tlo lemogwa gore mehuta ye mebedi ya phatose (pathos) le masetlapelo (tragedy) (kgopolo ye e ka se hlalošwe fa ka ge e se e lebane le maikemišetšo a nyakišišo ye) e lebana le moko wa ditaba wa go nepiša kgegeo (e theilwe godimo ga 'mošomo wa go swaolla' bophelong) (Muecke, 1982: 4) yeo e godišago goba e tšwetšago pele ditiragalo tša sengwalo sa mohuta wo (wa phatose). Ka go realo go yo akaretša ditiragalo tša tshekatshelo ya teori ya phatose ka boripana gore di tle di thuše kwešišo ya kgopolo ye ka tshwanelo. 
Ge a hlaloša se phatose e lego sona Baldick (1990: 163) o fo re ke sengwalo sa go ba le maikutlo a manyami le kwelobohloko '... a literary work or passage within it appealing especially to our feelings of sorrow, pity and compassionate sympathy'. Ke ka mokgwa woo Murfin le Ray (2009: 372) ba rego phatose ka Segerike e šupa khuduego, tlaišego goba masetlapelo, boleng ka gare ga sengwalo, bjo bo dirago gore setšhaba (mmadi / mmogedi) se itemogele manyami le kwelobohloko. Ka go realo phatose e fapana le masetlapelo ka gore maikemišetšo a moanegwagolo wa yona ga a kgone go tšwelela gabotse bjalo ka wa masetlapelo. Fa go bolelwa ka ditiragalo tša diphetogo tšeo di tlišago masetlapelo a khuduego ye kgolo go ba lapa le go karolo ya setšhabeng. Go thekga taba ye Oele (2007: 142-143) yena o no re phatose e lebane le go kwešwa bohloko ka go fetišiša. Ka mantšu a mangwe go kwešwa bohloko ga ntshe ga go bolelege, go šiiša namana tša mmele. Ke ka tsela yeo Solivan (1998:48) yena a rego phatose ke mohuta wa masetlapelo ao a sa amanego le go iketla, efela a amanago ka kudu le matshwenyego gammogo le mahlomola a magologolo.

Ge a tšwetša ditaba tše pele, Draper (1980: 160) o re phatose e tšweletša moanegwa wa yona bjalo ka motho yo a beetšwego thokwana. O re:

The root idea of pathos is the exclusion of an individual on our level from a social group to which he is trying to belong.

Go mmeela thokwana (exclusion of an individual) ga moanegwa yoo ke gona go godišago kgakanego le tlabego (Groenewald, 1993: 61). Go ya ka Oele (2007: 75), ditiragalo tša phatose di hlolwa ke dilo tše mmalwa, go etša diphetogo mo bophelong. Ge a mo amogetša kgopolong yeo, Potloane (1994: 81) o re e ka hlolwa ke dilo tša go swana le letšhogo le kgakanego mo bophelong, lenyalo la go hloka motheo, la go tekateka, moo motho a phelago a nnoši mola a nyetše molekane, ba swanetšego go ba mmogo mathateng le bohloking bjo bo itšego.

Ge a hlatsela taba ya diphetogo tšeo Solivan (1998: 104) o no re, phatose e ka swantšhwa le leeto la ge motho mo tseleng ya bophelo bja gagwe a iphahla ka lefego mo boteeng bja katanelo ya tumelo ya setšhaba go fihlelela diphetogo tša go amana le kwelobohloko ya go gatelelwa ka mokgwa wa go phethagala go ya ka modiro wa tlhago (Moyamokgethwa). 
Ge a godiša thalošo ya phatose go iša pele le pele Baldick (1990: 61) o bolela gore kgopolo ye e ka lebanywa le lehu la moanegwa wa maemo a mehleng. Lehu leo e lego lona le tlišago ditlaišego tše dikgolo tša ditho tša ba lapa tšeo di ka amago le bophelo bja karolo ya setšhaba.

Go ka fo rungwa ka go re phatose e akaretša bohloko bjo bogolo bjo motho a bo kwešwago ke ditlamorago tša bophelo go swana le setšo, histori, politiki le leago (Delius, 1983: 159-163) ka gobane '... pathos has a close relation to the sensational reflex of tears' (Draper, 1980: 160). Ka mabaka a mangwe, phatose e hlolwa gantši le ke ge motho a phela ka gare ga letšhogo le kgakanego mo bophelong. Ge motho a phela bophelo bja go hlakahlakana ka mehla, mafelelong o ikhwetša a le ka gare ga bophelo bja matshwenyego ao a fetago le a lehu, ao a ka hlalošwago go ba manyami le kwelobohloko ye kgolo (Oele, 2007: 192).

Ka fao ge, go ka gatelelwa ka go phetha ditaba tša bohlokwa bja phatose, e lego masetlapelo a a felelago ka kwelobohloko ya ba lapa le go karolo ya bophelo bja setšhaba ka mantšu a Holman (1972: 384) ge a re phatose ke tiragalo ye e itšego ka gare ga kanegelo/terama yeo e tsošago maikutlo a kwelobohloko ka gare ga mafahla a batho ba ba rilego le bona babadi/babogedi ka bobona.

Bjale ditiragalo tša phatose di tlo hlalošwa ge di lebane le kwelobohloko le manyami a magolo a go nyalanego le lehu la molwantšhwa yoo ba lapa le karolo ya setšhaba le mmadi ba ikgwarantšego le yena. Moanegwa yoo o welwa ke matshwenyego a magolo ka kudu go feta moanegwathwadi wa masetlapelo. Molwantšhwa yoo ke yo bolo; ke ka fao a ratwago ke bontši bja setšhaba ka lebaka la gona go loka goo ga gagwe; ebile kwelobohloko ya bona (karolo ya setšhaba) e feta le kwelobohloko ya ge go hlagile lehu. Ditaba tšeo di tlo hlalošwa ka tshwanelo ge go sekasekwa moanegwa yoo gona mo taodišwaneng ye.

Mohlala wo mobotse mo dingwalong tša Sepedi wa kanegelo ya phatose ke wa kanegelo ya Tladi wa Dikgati. Ka lebaka la gore ditiragalo tša phatose di fela di hlakahlakanywa bobading le bosekaseking bja masetlapelo, go bohlokwa go fa thalošo ye e tseneletšego ya ditiragalo tša mohuta wo wa sengwalo sa phatose, ka go sekaseka kanegelo yeo ya go ngwalwa ke Phatudi ka ngwaga wa 1958 gore go tle go be le lesedi tharollong ya kgakanego le tlabego tša go lebana le go ba gona ga kwelobohloko dingwalong tša mohuta wo wa masetlapelo le wa phatose.

Ge Tladi wa Dikgati e eyo ahlaahlwa, go gatelela thulaganyo ya tharollo ya bothata bjo, go yo hlokomelwa lenaneo le le latelago la ka mo tlase la go hlagiša 
pharologantšho ya maatla a kwelobohloko le manyami gape le go tšweletša ditsela tša go godiša morero wa sengwalong sa mohuta wo.
(a) Kakaretšo ya kanegelo ye
(b) Molwantšhwa wa kanegelo ye
(c) Ditiragalo tša mohutangwalo wo
(d) Ke ka lebaka la eng kanegelo ye e bitšwa ya phatose?
(e) Morero wa kanegelo ye
(f) Dithekniki tša thulaganyo
(g) Thumo

\section{Kakaretšo ya kanegelo ye}

Phatudi o anega ditiragalo tša kanegelo ye ya phatose ya Tladi wa Dikgati ka ga bophelo bja Tladi, ngwana ebile e le morwa wa Dikgati.

Ka go realo morero wa kanegelo ye ya phatose o nepiša bophelo bja manyami a magolo a lehu ka tsela ya go tlabega le go gakanega. Kgopolo ye e thekgwa ke ge Phatudi a anega merero ya bophelo bja Tladi, morwa wa Dikgati, yoo a golelago lapeng la Dikgati gammogo le morwarragwe, Monyane. Monyane ke morwa wa Dikgati yo mogolo wa segwahlana (yo a otlilwego ke thago ka ge e be e le segole). Tladi ke morwa yo monnyane wa Dikgati yoo a phetšego gabotse mmeleng.

Ge nako ya lebollo e fihla, Monyane o palelwa ke go ya komeng, ka lebaka la bogole bja gagwe. Ka mokgwa woo Tladi ga a dumelelwa go bolla pele ga mogolwagwe, Monyane. Tladi o išwa ga rakgolwagwe moo a go golela gona, ka lebaka la bogwahlana bja mogolwagwe, Monyane.

Ka go hlompha mogolwagwe, Monyane, le setšo Tladi o tšhabela komeng mefatong e šele, kua gaMasemola, ka ge a se a swanela go bolla ga gabo pele ga mogolwagwe. Madimabe Tladi o hlokofalela ka molotong. Tlhokofalo yeo ya Tladi e tsoša tlabego le kgakanego ka tsela ya kwelobohloko ye kgolo go karolo ya setšhaba (gagolo ba meloko le metswalle ya gagwe).

Ka fao ge, lebaka le legolo la go akaretša ditiragalo tša padi ye, ke go gatelela maatla a sererwa (topic) gammogo le ka fao sererwa se ikgethela le go laola baanegwa ba teori ya diteng (content) ka gona. Bjale go yo bontšhwa modiro woo wa sererwa le wa baanegwa (ba diteng) mo pading ya Phatudi. 


\section{Sererwa sa phatose}

Ge a hlaloša kgopolo ya sererwa (topic) Mojalefa (1994:20) o re ke ge ditiragalo di latelana ebile di tlemaganywa ke selo (se tee), seo se kgethelago mongwadi ditiragalo tše a tlogo ngwala ka tšona. Ke ka fao Marggraff (1994:61) a hlalošago sererwa ka go re ke kgopolokgolo ya mafelelo yeo mmadi a e fihleletšego mo sengwalong. Ke go re, ke ge mmadi a fihlile magomong a mafelelofelelo ao a ka se sa kgonago go tšwela pele go akaretša ditaba tšeo a ratago go di akaretša.

Sererwa sa kanegelo ye ya Phatudi se lebane le ge Tladi a ganetšwa go ya komeng ka nako e tee le Monyane, mogolwagwe; ke go re, ka boripana, Tladi o ganetšwa go ya komeng.

Sererwa se se ka akaretšwa ka go re ge nako ya go ya komeng e fihla, Monyane o šitwa go ya molotong ka lebaka la bogwahla bja gagwe. Ka tsela yeo Tladi o ganetšwa go bolla pele ga Monyane. Ka lona lebaka leo Tladi o išwa ga rakgolwagwe moo a go golela gona, ka lebaka la bogole bja mogolwagwe.

Ka lebaka la go hlompha mogolwagwe, le tshepetšo ya melao ya setšo, Tladi o tšhabela komeng mefatong e šele, gaMasemola, ka lebaka la ge a se a swanela go bolla ga gabo pele ga mogolwagwe go ya ka melao yeo ya bogologo ya segagabo. Madimabe Tladi o hlokofalela ka molotong. Tlhokofalo yeo ya Tladi e tsoša tlabego le kgakanego ka tsela ya kwelobohloko ye kgolo go ba lapa labo le go karolo ye e rilego ya setšhaba (ba motse wa gabo).

Ge kakaretšo ya sererwa se e lekodišišwa, go lemogwa gore se ipopile/theilwe ka dikarolwana/dikokwane tše pedi tše kgolo, e lego:

- Go ganetšwa go ya komeng ga Tladi

- Go hlokofalelwa ka komeng ga Tladi

Bjale go yo akaretšwa kokwane (moko) ye bohlokwa ya dikarolwana tše pedi tšeo tša sererwa. 
Ge nako ya go bolla ga Monyane e fihla, o šitwa go ya lebollong ka lebaka la bogole bja gagwe. Ge lebaka la go welwa ga Tladi le fihla, le gona Tladi o šitwa go ya mphatong ka lebaka la (a) bogwahlana bja mogolwagwe, Monyane, gammogo le gore (b) o hlompha mogolwagwe, Monyane, le (c) tshepedišo yeo ya melao ya segagabo malebana le ditaba tša mohuta wo. Ke ona mabaka a mararo a magolo ao a dirago gore Tladi a paledišwe go phetha merero ya bonna ka tsela ya lebollo ka lebaka la bogole bja Monyane.

\section{Go hlokofalelwa ka komeng ga Tladi}

Dikgati, ka go lemoga bothata bjoo bja morwagwe Monyane, o napile o iša Tladi, ga rakgolwagwe moo a go golela gona. Ge koma e etšwa moo gaMasemola, ka lebaka la go šitwa ke maemo a thago (bogolo), e lego gore bjale ke thakakoma, o šitwa go tšwela pele go emela mogolwago gore a bolle pele ga gagwe, go ya ka taelo ya melao ya bogologolo. Tladi o tšhabela komeng mefatong e šele (kua gaMasemola), ka lebaka la ge a se a swanela go bolla ga gabo pele ga mogolwagwe, go ya ka thulaganyo ya melao ya bogologo ya segagabo. Madimabe Tladi o hlokofalela ka komeng. Tlhokofalo yeo ya gagwe e tsoša tlabego le kgakanego ka tsela ya kwelobohloko ye kgolo go ba bangwe ba batho ba setšhaba (ba lapa labo).

\section{Mongangiši wa phatose}

Mojalefa (1995:6) o bolela gore moanegwa wa teori ya diteng ke motho wa sengwalwa, le ge e le gore le dilo e ka ba moanegwa. Go ka thwe ge go hlalošwa kgopolo yeo, gwa thwe ke moraloki go phala gore ke motho, ka gobane ge go bolelwa ka moraloki go bolelwa le ka selo eupša e sego motho fela (Bal, 1980: 14-15). Moanegwa yoo o bitšwa mongangišwa (kind-hearted person) goba mongangiši (quarrel-some person). Mongangišwa ke moanegwa yo mogolo yoo a ngangišetšwago go loka mola ka lehlakoreng le lengwe mongangiši e le moanegwa wa senganga, wa go se ratege, yoo a sa dirego go loka ka dinako tšohle (Mojalefa, 1995: 6).

Bjale ge go sekasekwa kanegelo ye ya Phatudi ya phatose ya go bitšwa Tladi wa Dikgati, go lemogwa gore moanegwa yoo ke mongangišwa, e lego Tladi ka gobane 
o dira go loka; ka fao mmadi (le karolo ya setšhaba) o a mo rata le go mo kwela bohloko ka ge a se na le phošo le gateetee. Ditaba tša go tšhabela komeng ga se phošo ya gagwe. Ke phošo ya thago (bogwahlana bja mogolwagwe gammogo le melao ya segagabo - o se bolle pele ga mogolwago). Ka go realo o ngangišwa ke thago le melao ya setšo (go thwe moanegwa ga se motho fela le selo e ka ba moanegwa) gore a bolle ka nako ya gagwe yeo e mo lekanego go swana le dithaka tša gagwe.

\section{Molwantšhwa wa kanegelo ye}

Mojalefa (1995: 20) o re baanegwathwadi ba thulaganyo ke molwantšhwa, molwantšhi le mohlohleletši. Mo nyakišišong ye go yo bolelwa ka molwantšhwa ka ge bao ba bangwe ba se na tebanyo ya yona. Ka go realo, go ya ka Mojalefa ka godimo, moanegwa yoo ke yo mogolo yoo karolo ye kgolo ya sengwalwa e bolelago ka yena. Go ya ka Abrams (1988: 139) ke motho yoo kgahlego ya babadi e letšego go yena. Gape motho yoo babadi ba swanetše go ikgweranya le yena. Moanegwa yoo a ka Iwantšhetšwa go loka goba go se loke ga gagwe. Godimo ga moo baanegwa ba thulaganyo ba na le mošomo - mošomo woo ke wa boswantšhi (Groenewald, 1993: 35).

Moanegwa wa kanegelo ya mohuta wo wa phatose ke motho yo mošomo wa gagwe e lego go tšweletša bophelo bjo bo phelwago ke motho wa go se be le sa gagwe (modiitšana). Ke moanegwa yo a sa amanego felo le maemo ao a phagamego setšhabeng seo a phelago go sona. Ka go realo moanegwa wa phatose o nepiša (emela) bophelo: go tlabega le go gakanega.

Go wa ga gagwe (molwantšhwa) mo bophelong ga go ame setšhaba ka moka, ka ge e no ba motho fela, yo a se nago maemo a a rilego. Le ge go le bjalo, ge a wele, ba lapa le karolo ya setšhaba gammogo le bona babadi ba no mo kwela bohloko, ka lebaka la go emela go loka (e lego mošomo/modiro wa gagwe) goo ga gagwe. Go realo go ra gore mogale yo wa sengwalo sa phatose ga a na mafokodi ao a ka mo amanyago le diphošo tša go lebana le lehu la gagwe, goba ona masetlapelo a go ba le maatla a go feta le lehu. Ge go bapetšwa thalošo ye le ditiragalo tša Tladi wa Ditkgati, go lemogwa se molwantšhwa, Tladi, a lego sona moanegwa wa kwelobohloko ye maatla. 
Ge Tladi wa Dikgati, bjalo ka molwantšhwa wa Tladi wa Dikgati, semelo sa gagwe se sekasekwa bjalo ka sengwalo sa kanegelo ya phatose, seo se laolwago ke moko wa ditaba, se tlo lebanywa le morero (design) woo Mojalefa (1993: 27) a o bitšago lenaneotlhophangwalwa, woo o hlalošago gore moanegwa yo ke molwantšhwa wa phatose, yoo e lego:

- Motho wa maemo a fase; ke go re motho wa mehleng.

- Ga a na bofokodi; ke moloki wa go hloka bošaedi.

- Le ge a se na bofokodi o a hlokofala ka lebaka la kgakanego le tlabego.

- O kwelwa bohloko ge a hlokofala ka gore ke motho wa go loka.

Bjale go yo tsitsinkelwa morero wa padinyana ye go ya ka dikokwane tše tše nne tša ka godimo tša moanegwa wa phatose go lebeletšwe gore molwantšhwa ke:

\section{Motho wa maemo a fase}

Moanegwathwadi wa sengwalo sa phatose o swanetše go ba motho wa maemo a a rilego a fase, e be mohlaki le mohloki. Ke go re ga a na maemo bophelong; ke motho wa go hloka sa gagwe. Ge a tiiša maemo ao, Phatudi o re:

Mmasefako le Mmapule ba be ba robala ka morago mathuding a ngwako woo go robalago mmagobona (letl.8).

le

'Ba akanya gore morwa' Dikgati le ge a be a eme ka maoto, a le dipotokwane, o be a sa le ngwana gobane le komeng o be a sa ba a hlwa a eya gona' (letl.33).

Legae la boTladi, ke la go hloka ka gobane le hlaelela madulo a maemo a godimo gammogo le ditlabakelo tše dingwe tša go bontšha gore ke la mabaibai: 
Mmasefako le Mmapule ba be ba robala ka morago mathuding a ngwako..., tatago bona yena a robala ka ntlwaneng ka mafuri (ebile) go na le ngwakwana wa mollo (letl.8).

Godimo ga moo, go thwe Tladi e be:

e sa le ngwana gobane le komeng o be a sa ba a hlwa (letl.33).

Ge motho e sa le ngwana ga a na ditseka, ka go realo ga a na sa gagwe - bophelo bja gagwe bo itshamile ka bja ba bangwe (batswadi ba gagwe).

\section{Ga a na bofokodi}

Go kgonthiša go hloka bofokodi ga Tladi, Phatudi o no re:

Na koma e fetola matswalo le bogolo bja motho? Aowa, ga e fetole selo. Monyane ke mogolle. Go tla no ba bjalo ka mehla le ge re ka ya mphatong gotee (letl.46).

Tladi o gatelela gore yena ga a na le phošo - phošo o e rweša melao ya setšo:

Na koma e fetola matswalo le bogolo bja motho? Aowa, ga e fetole selo. (letl.46)

Ka lehlakoreng le lengwe mongwadi o kwana le Tladi ka go tšweletša mafokodi a a rilego a mogolwagwe Monyane, ka gore o bolela gore Monyane o na le mafokodi ka go re:

'Monyane ke segwahla. Ke mofokodi'.

'O a rereša. Ke yo boima Monyane ruri,' go bolela yo mongwe (letl. 19).

Bofokodi bja Monyane bo lebanywa le bolwetši le bogwahlana bja gagwe go ya ka fao bo hlalošwago ke baanegwa ba bangwe ka gona. Ge moanegwa yo a phelago le go tseba dilo ka moka tša bophelo bja Monyane a re ke segole; ke go re o na le 
mafokodi bophelong bja gagwe, mmadi o amogela ditaba tšeo ka botlalo ka gobane o tseba Monyane go feta le mongwadi.

\section{O a hlokofala (le ge a se na mafokodi)}

Phatudi o re Tladi o ya fase le ge a se na mafokodi a a rilego ka gobane o a hlokofala. O re:

Matwetwe a lemoga gore molwetši o dikologwa ke phiri gomme a ba a lemoga gape gore Dikgati o tsenwe ke maseme ka maatla (let.55).

le

Jo-nna-jo! Dikgati a hlaba mokgoši gobane o bone ka mahlo... Banna ba ile ge ba ekwa sello sa Dikgati ba thoma ka pela go hlaba mašata le go opela go tima le go thibela gore sello sa Dikgati se se kwale ka ge e le mokgwa wa komeng go dira bjalo (letl.55).

Dipolelo tše di gatelela lehu; ke go re le ge Tladi a se na mafokodi - mafokodi e le a tlhago, o feleletše a le moletemohlaelathupa. Taba ye e hlola kwelobohloko le manyami a magolo mafahleng a batho ka gobane (bona) ba a gakanega le go tlabega ka lebaka la tiragalo yeo ye mpe/manyami ya go hlagela Tladi.

\section{Mmadi o mo kwelwa bohloko}

Go tiiša kgopolo ya batho ya go kwela Tladi bohloko ge a tšewa ke phiri, mongwadi o re:

Kgwedi ya boraro e be e šetše e balamile gomme go šetše fela dibeke di le tharo gomme boTladi ba fologa thabeng. Dikgati bjalo ka mehleng a leba morotong go yo bona Tladi. A hwetša Tladi le ba bangwe ba le balwetši kudu! Ba palelwa ruriruri gomme ba šetše ba šala morotong ge koma e eya go fula (letl.53). 
Malekutu le Kunupi, bakgomana ba bagolo gaMasemola, bona beng ba koma, mmogo le Dikgati ba fihla ka pele go matwetwe.

'Babinaphiri' go realo ngaka, 'naka e šitile moletši'. Sefahlego sa gagwe se senyegile (letl.55).

Maemo a bolwetši bja Tladi ka mphatong, a ipopile sethakgodi, e lego tiragalo ya go hlola thulano (ye kgolo) ya manyami. Ka go realo bolwetši bo fetoga semamathane (moromiwa) sa lehu leo le tlelago Tladi:

... 'naka e šitile moletši'. Sefahlego sa gagwe se senyegile.(letl.55).

Ka fao, ditiragalo tšeo di bakela ba lapa le batho ba bangwe gammogo le yena mmadi go ba le kwelobohloko ye kgolo dipelong tša bona.

\section{Ditiragalo tša mohutangwalo wo}

Go lemogilwe gore ditiragalo tša sengwalo sa phatose, ge di bapetšwa le tšeo tša masetlapelo, di lebanywa le go hlokofala ga moanegwa wa mehleng, wa go hloka maemo setšhabeng. Gantši ditiragalo tšeo di ama lapa le meloko ya lona (bjalo ka lapa la Dikgati le bana ba gagwe). Godimo ga moo, se bohlokwa ka ditiragalo tšeo ke kokwane ya go loka. Ke go re ditiragalo di theilwe godimo ga toko/bolo (Tladi o lokile ka gore o na le thompho).

Ditiragalo tša mohutangwalo wo di amana ka kudu le taba ya go beelwa thokwana ga moanegwagolo/molwantšhwa (Tladi o išwa gaMasemola) eupša le ge go le bjalo, lapa le meloko ba ikgweranya le gona go itswalanya le yena ka lebaka la kwelobohloko; ke yona taba yeo e hlolago kgakanego le tlabego (lehu la Tladi).

Ge ditiragalo tšeo tša phatose, e lego tša Tladi wa Dikgati, di lekolwa, go yo hlokomelwa:

- Ditiragalo tša lehu la moanegwa wa mehleng

- Ditiragalo tša go ama lapa le meloko 
- Ditiragalo bjalo ka kokwane ya go loka

- Ditiragalo tša go hloka bofokodi ebile di tsošago maikutlo a kwelobohloko

- Ditiragalo tša go makatša tša go tšweletša dinnete tša bophelo

- Ditiragalo tša go šiiša namana tša mmele ebile tša go tsoša kwelobohloko ye kgolo

Bjale go yo tsinkela dikokwane tšeo tša ditiragalo tša sengwalo sa phatose ka go di nepiša le padinyana ya Tladi wa Dikgati.

\section{Ditiragalo tša lehu la moanegwa wa mehleng}

Ge a hlaloša ditiragalo tša moanegwa yoo wa mehleng wa go hloka le sa gagwe, mongwadi o re:

Ka letšatši le lengwe, maru a phefo a tletše legodimo gomme go tonya, too! ka nako ya ge koma e goroga e etšwa go fula, Tladi a golelwa ke bolwetši ka kudu. Matwetwe a lemoga gore molwetši o dikologwa ke phiri gomme a ba a lemoga gape gore Dikgati o tsenwe ke maseme ka maatla (let.55).

le

'Babinaphiri' go realo ngaka, 'naka e šitile moletši.' Sefahlego sa gagwe se senyegile (let. 55).

Ditsopolwana tše tša ka godimo di hlaloša lehu la Tladi ka batho ba babedi, e lego mongwadi le ngaka. Phatudi o diriša batho ba babedi bao go anega ditiragalo tšeo tša lehu la Tladi go gatelela gore ka nnete Tladi o tšerwe ke phiri. Ka tsela yeo, palo ya bobedi (ke go re lehu le begwa ke batho ba bantši (babedi), e sego motho o tee) yeo, e godiša kwelobohloko, masetlapelo le manyami a magolo mafahleng a batho le go mmadi. Ka go realo go ka thwe palo yeo (ya bobedi) e tiiša lehu la Tladi, moanegwa wa mehleng, wa go ithakela - tatagwe ga a na sa gagwe. Ge a (Dikgati) 
ka be a na le sa gagwe, mohlomongwe o be a tla kgona go phološa morwagwe dinaleng tša lehu ka go hwetša ngaka ye nngwe ya go mo thuša bolwetšing bjoo.

\section{Ditiragalo tša go ama lapa le meloko}

Mongwadi ge a hlaloša ditaba tšeo o re:

Dikgati o be a dutše setulwaneng. Monnamogolo, Mogalatšane, a dutše fase phateng, a itshetlegile ka lebota; Tladi a le thoko ya gagwe ka go la go ja gomme Mohlehle, mmagoTladi, mogatša' Dikgati, a khunamile go lebana le monna wa gagwe ka le letshegadi.

Mogalatšane a bula molomo:

'Malope!'

'Mogale!'

'Ke tlile mo ka taba'

'Mahlako o a kwa na? go realo Dikgati...

Ke tlile ka molato wa yo morwago, Tladi! Maabane o tlile a lla mo go nna. O re, thaka tša gagwe di mo šiile, di ile mphatong gomme le yena o rata go ya gona... Tladi o re: 'ke tennwe ke bošoboro ke nyaka go ya komeng bjalo ka ba bangwe. Ke šetše ke nnoši gomme batho ba ntshega'... 'Malope, ke ya di kwa ditaba tše' go realo Mohlehle (letl.44).

Go ya komeng ga Tladi go bolelwa ke ba lapa. Ke go re ditaba tša go rata go wela ga Tladi di šilwa megopolong ya ba lapa. Go rulaganya ditaba bjalo, Phatudi o rato gatelela bogale le bošoro bja tiragalo ye e tlago ya masetlapelo, e lego lehu la Tladi, yeo e tlogo wela ba lapa leo; ke gore mongwadi o šomiša thulaganyo ya thekniki ya tekolapejana (go bolela ka ditiragalo tšeo di tlogo direga kua pele).

\section{Ditiragalo bjalo ka kokwane ya go loka}

Ge Phatudi a hlaloša ditiragalo tšeo bjalo ka kokwane ya go loka, o no re:

'Fela ga di ye ka tsela gobane Monyane a ka se thake le Tladi. Sesotho se a gana le tshwanelo ga se yona go dira gore bana ba motho ba nyatšane. 
Tladi a se ke a taboša Monyane mahlo eupša ga a mo hlomphe, ke kwana kudu le mantšu a rakgoloagwe' (letl.44).

Ntshetshere ya go ya molotong ga Tladi, ga se phošo ebile Tladi ga a nyatše Monyane; o a mo hlompha. Bothata bo hlolwa ke tshepedišo ya melao ya setšo:

Sesotho se a gana le tshwanelo ga se yona go dira gore bana ba motho ba nyatšane.

Tšeo di bolelwago ka godimo ga di dire gore go thwe Tladi ga se a loka. Go se loke go lebane le bogologolo. Ke go re ditiragalo di begwa ka mokgwa wa lehlakore la go se loke go gatelela lehlakore la go loka.

\section{Ditiragalo tša go hloka bofokodi ebile tša go tsoša maikutlo a kwelobohloko}

Ge a kgonthiša ditiragalo tšeo, mongwadi, Phatudi, o fo re:

Ke molao wa go fahlwa, wa go leka go thibela motho go ba monna mola o sa kgone go mo thibela go gola! Ke molao wa go senya sebaka fela. Le gona mphato wona wo o lebane nna, e sego Monyane (letl.46).

le

Tladi a golelwa ke bolwetši ka kudu. Matwetwe a lemoga gore molwetši o dikologwa ke phiri gomme a be a lemoga gape gore Dikgati o tsenwe ke maseme (letl.55).

Tiragalo ya setsopolwa sa mathomo e gatelela gore ke molao wa setšo woo o nago le phošo ka go ganetša Tladi ge a eya molotong, eupša e sego Tladi ka gobane nako ya gagwe ya lebollo e fihlile. Go realo go hlalošwa go hloka bofokodi ga Tladi.

Ka lehlakoreng le lengwe tiragalo ya setsopolwa sa bobedi yona e gatelela ka fao Tladi a tlaišegago ge a le kua komeng ka lebaka la bolwetši bjo bo mo hlasetšego; ka go realo e tsoša maikutlo le kwelobohloko. 


\section{Ditiragalo tša go makatša tša go tšweletša dinnete tša bophelo}

Tladi o gapeletša gore a lokollwe go yo bolla ka gobane ge a ka se bolle o tla fetoga lešoba; yona taba yeo e tlago go ba semaka bophelong bja gagwe. O re:

'Na koma e fetola matswalo le bogolo bja motho? Aowa, ga e fetole selo. Monyane ke mogolle. Go tla no ba bjalo ka mehla le ge re ka ya mphatong gotee' (letl.46).

O tšwela pele go nyatša le go nyefola setšo ka gore o re se a mo makatša ka fao se lego ka gona:

'Ke molao wa go fahlwa, wa go leka go thibela motho go ba monna mola o sa kgone go mo thibela go gola! Ke molao wa go senya sebaka fela. Le gona mphato wona wo o lebane nna, e sego Monyane' (letl.46).

Ka polelo ye mongwadi o dirišitše sekapolelo sa kganetši go gatelela maemo a Tladi a go ngangabala. Mampuru (1991:80) o hlaloša kganetši gore ke:

Go tšweletša kganetšano le phapanyo mo go hlalošeng seo go bolelwago ka sona. Kganetšano ye e tšwelela ka mokgwa wa go bapetša dilo tšeo di fapanago. Ka papetšo ye mmadi o kgona go bona kganetšano ya tšona le go kwešiša kgopolo yeo e tšweletšwago.

Ka fao mongwadi o diriša papetšo go fapantšha dilo (dikgopolo) tše pedi, e lego molao (tshepedišo ya setšo) le Tladi (ge a ganetša molao woo wa bogologolo go phethagatšwa). Go ka thwe ka go realo Phatudi o thulanya dikgopolo tša bogologolo le tša sebjalebjale, e lego dikgopolo tše mpsha tša Tladi malebana le tshepedišo ya setšo mererong ya lebollo.

\section{Ditiragalo tša go šiiša namana tša mmele ebile tša go tsoša kwelobohloko ye kgolo}

Ge a kgonthiša ditiragalo tšeo tša go garola matswalo, Phatudi o no re: 
Jo-nna-jo! Dikgati a hlaba mokgoši gobane o bone ka mahlo... Banna ba ile ge ba ekwa sello sa Dikgati ba thoma ka pela go hlaba mašata le go opela go tima le go thibela gore sello sa Dikgati se se kwale ka ge e le mokgwa wa komeng go dira bjalo (letl.55).

Ge motho wa monna a šetše a hlaba phooko (go lla), mola go thwe monna ke nku o llela teng, go bontšhwa/gatelelwa bošoro le bogale bja tiragalo yeo ya mahlomola a magolo, ka gobane monna o a kgotlelela ka gore ga se ngwana goba mosadi. Bjale ge Dikgati a šetše a šitwa go kgotlelela, go laetša gore tiragalo ye bjalo e kweša bohloko ka kudu le go feta. Ka fao tiragalo yeo e tshotshoma ka manyami le kwelobohloko ye kgolo yeo e amago le ba lapa le karolo ya setšhaba.

\section{Ke ka lebaka la eng kanegelo ye e bitšwa ya phatose?}

Taba ya pele: Gantši kanegelo ya phatose e na le moanegwagolo wa maemo a fase. Go tlo tsopolwa mohlala wa Tladi wa Dikgati bjalo ka molwantšhwa wa maemo a mehleng.

Bjalo ka ge e be e le marega, phefo e gaketše gomme mmu o ke o kolobile. Nao tša Tladi tša phaka tša hwa gomme go sepela ya eba go šita kgang. Mo mmeleng, Tladi a se kwe phefo kudu ka lebaka la mantabulana a a bego a ena le ona. Monnamogolo yena $o$ be a tsene jaseng ya matšoni gomme a tšhahlanketše ka dimphašane (let.28).

le

Ba akanya gore morwa' Dikgati le ge a be a eme ka maoto, a le dipotokwane, o be a sa le ngwana gobane le komeng o be a sa ba a hlwa a eya gona (let. 33).

Polelo yeo e gatelela maemo a mehleng (go hloka sa gagwe) a Tladi:

Nao tša Tladi tša phaka tša hwa gomme go sepela ya eba go šita kgang. Mo mmeleng, Tladi a se kwe phefo kudu ka lebaka la mantabulana a a bego a ena le ona. 
Taba ya bobedi: Kanegelo ya phatose e na le molwantšhwa wa go hloka mafokodi. Go kgonthiša ditaba tše mongwadi o re:

Ke molao wa go fahlwa wa go leka go thibela motho go ba monna mola o sa kgone go mo thibela go gola. Ke molao wa go senya sebaka fela. Le gona mphato wona wo o lebane le nna, e sego Monyane (let. 46)

Go ka thwe, Tladi o hloka mafokodi ka gobane diphošo tše o ka rego o a di dira, di lebane le tshepedišo ya melao ya setšo, e sego bofokodi go tšwa lehlakoreng la gagwe.

Taba ya boraro: Ditiragalo tša kanegelo ya phatose di nepiša bophelo: go tlabega le go gakanega.

Mongwadi, Phatudi, o fo re:

Tladi a golelwa ke bolwetši ka kudu. Matwetwe a lemoga gore molwetši o dikologwa ke phiri gomme a be a lemoga gape gore Dikgati o tsenwe ke maseme (letl.55).

Mongwadi o diriša kgakanego le tlabego bjalo ka kokwane ye bohlokwa ya bophelo bja moanegwa wa sengwalo sa phatose ka gobane di godiša morero wa mohutangwalo wo. Kgakanego le tlabego tšeo di hlagišwa gagolo gape ka mokgwa wo, gore di bonagale le go godišwa gabotse:

Jo-nna-jo! Dikgati a hlaba mokgoši gobane o bone ka mahlo... Banna ba ile ge ba ekwa sello sa Dikgati ba thoma ka pela go hlaba mašata le go opela go tima le go thibela gore sello sa Dikgati se se kwale ka ge e le mokgwa wa komeng go dira bjalo (letl.55).

\section{Morero wa kanegelo ye}

Padi ye ya Phatudi e theilwe godimo ga ditaba tša bogologolo/kgale. Phatudi o hlaloša ditiragalo tša lebollo. Ditaba tšeo ke tša setšo. Moko wa ditaba o lebane le tumelo ya go re motho a ka se ete mogolwagwe pele mererong ya bophelo, (goba bophelo bja tlalelo le kgakanego): e ka ba mošemane goba ngwanenyana. 
Ke ka lebaka leo Tladi a ganetšwago go ya komeng ka nako e tee le Monyane, mogolwagwe. Monyane o šiilwe ke dithaka tša gagwe ka lebaka la bolwetši/bogole. Tladi ga a kwešiše ge a se a swanela go ya komeng le dithaka tša gagwe. Ke go re, o tlo ya komeng le moroto wo o latelago. Mongwadi o diriša kgakanego go godiša moko wa ditaba tša padi ye. Ge a kgonthiša kgakanego yeo Phatudi o fo re:

Jo-nna-jo! Dikgati a hlaba mokgoši gobane o bone ka mahlo... Banna ba ile ge ba ekwa sello sa Dikgati ba thoma ka pela go hlaba mašata le go opela go tima le go thibela gore sello sa Dikgati se se kwale ka ge e le mokgwa wa komeng go dira bjalo (letl.55).

\section{Dithekniki tša thulaganyo}

Setsopolwa se sa ka mo godimo, se yo hlalošwa go thuša go bonagatša mediro ya thekniki. Taba ye e šetše e kgwathilwe gannyane ka godimo. Ka thekniki go gatelelwa gore ke selo seo se bonwago seo se tlogo kgona go šomišwa go tšweletša seo se bolelwago. Gomme ka go šomiša thekniki, mongwadi o kgona go tšweletša tebanyo ya gagwe (Kerkhoff, 1962: 16). Ke ka fao go thwego ke seo mongwadi a se bolelago ka go ngwala, le ka wona mokgwa woo a se boletšego ka gona ka go se ngwala. Ke ka tsela yeo go ka thwego ke mokgwa woo ka wona mongwadi a tšwetšago pele moko wa ditaba (Mojalefa, 1997: 17) le Marshal McLuthan (Lazarus \& Smith, 1983: 289). Ka go realo thekniki e na le mediro ye e rilego, e lego (a) go gatelela kgopolo, (b) go godiša kgopolo, (c) go tiiša kgopolo ye e itšego le (d) go lebantšha kgopolo yeo le moko wa ditaba.

Bjale go yo šetšwa mediro yeo ya thekniki mo kanegelong ya phatose ya Tladi wa Dikgati, e lego.

- go gatelela kgopolo,

- go godiša kgopolo,

- go tiiša kgopolo ye e itšego le

- go lebantšha kgopolo yeo le moko wa ditaba.

\section{-}




\section{Go gatelela kgopolo}

Ge go balwa lelahlelwa la 'Jo-nna-jo!' mo setsopolweng sa ka godimo go lemogwa ka fao, Phatudi a ratago gatelela kgopolo ya bošoro le bogale bja lehu ka go diriša sello sa motho wa monna, Dikgati, go hlaloša maemo a mabe a tiragalo yeo, ka gobane go thwe monna ke nku o llela teng. Ka tsela yeo, ka thekniki yeo ya kgatelelo ya kgopolo, mongwadi o hlaloša ditiragalo gore di bonagale gabotse mahlong a mmadi gore di tle di kwešišege gabonolo le ke bohle (setšhaba).

\section{Go godiša kgopolo}

Bjale ge go balwa:

Dikgati a hlaba mokgoši...

gona ka mo setsopolweng sa ka godimo, go lemogwa gape gore Phatudi mo o nyaka go godiša kgopolo yela ya go hlokofala ga Tladi ka mphatong. Go hlaba mokgoši ke tsela ye nngwe ye šoro ya go bontšha polelo ya sello sa manyami le masetlapelo a magolo, gagolo sa motho yo mogolo ka gobane ga go ke go bolelwa ka go hlaba mokgoši ga ngwana. Ka fao go hlaloša ditaba ka mokgwa wo, go lebane le go godiša kgopolo ye e rilego, e lego bogale le bošoro bja lehu ka tsela ya semaka le tlabego.

\section{Go tiiša kgopolo ye e itšego}

Mongwadi polelong ya setsopolwa sa ka godimo o re:

Banna ba ile ge ba ekwa sello sa Dikgati ba thoma ka pela go hlaba mašata le go opela...

Go hlaba mašata le go opelela godimo go tiiša kgopolo ye e itšego ya go re ke nnete Tladi o hlokofetša. Bjale baditi ba koma ba dira mašata le go opela (ga bodikana) ka maatla gore sello sa Dikgati se se tlo kwewa ke basadi le bana kua gae, (koma ke mmutla) ka gobane ge a ka kwewa o tlo ba a tlontlolotše seriti sa koma. Ge 
modikana a hlokofaletše komeng, tiragalo yeo ga e tsebišwe (le semeetseng) go fihlela koma e aloga.

\section{Go lebantšha kgopolo yeo le moko wa ditaba}

Bjale ge, ge go balwa gape:

Jo-nna-jo! Dikgati a hlaba mokgoši gobane o bone ka mahlo...

go tlo lemogwa gape gore 'Jo-nna-jo!' gammogo le ' ... a hlaba mokgoši ...' ke polelo ye e tšweletšago dikgopolo tše di lebanego le molaetša wa Phatudi, e lego bophelo bja manyami a magolo a lehu ka tsela ya go tlabega le go gakanega. Go thwe polelo yeo e nepiša morero wa mongwadi ka gobane ke ya sello se bogale sa manyami le masetlapelo se se tšwetšago pele tebanyo ya Phatudi ge a tlo ngwala kanegelo ye ya phatose.

Bjale pele ga ge go lekolwa morero wo wa phatose, go tlo rungwa ka go gatelela gore thulaganyo ya ditaba tša kanegelo ya mohutangwalo wo ga ya swanela go raragana (ka kudu) ka ge e lebane le kwelobohloko (ya karolo ya setšhaba). Ditaba tšeo di yo akaretšwa ka boripana go hlokometšwe ditiragalo tšeo tša kanegelo ya Tladi wa Dikgati.

Kanegelo ya Tladi wa Dikgati ge e lekolwa go lemogwa gore thulaganyo ya yona ga se ya raragana; ditiragalo di latelane go ya le ka fao di hlolanago/hlatlamanago ka gona. Ditaba di thoma mathomong, tša latelana bjalo go fihla mafelelong. Taba ye e dira gore sengwalo sa phatose se balege gabonolo ntle le ditšhitišo.

Kanegelo ye e arotšwe ka ditemana; mabakeng a mangwe mongwadi o fela a arola moela wa dikgopolo ka go šomiša dikarolwana tša go arola kanegelo ka dihlogwana tše lesometee gomme hlogwana ye nngwe le ye nngwe e na le leina la yona - e reetšwe leina, ebile e akaretša ditaba tša karolwana yeo e amegago. Go realo ke gore thulaganyo ye ya Tladi wa Dikgati ke yeo e kgotsofatšago dinyakwa tša sengwalo sa phatose. Ka gona go ka thwe e loketše babadi ka gore thulaganyo yeo e raranego e ka swariša babadi bothata ka ge e tlo ba e bolaya moya wa khuduego 
ye e letetšwego. Seo se bolela gore tatelano ye bonolo ya ditiragalo ke kokwane ye bohlokwa sengwalong sa phatose.

Bjale go yo lekolwa morero/molaetša woo, e lego tumelo ya go re motho a ka se ete mogolwagwe pele mererong ya bophelo (goba bophelo bja tlalelo le kgakanego), ka go o nepiša le ditsopolwa tšeo di o godišago gore o tle o bonagale gabotse ka go hlokomela dikokwane tše di latelago, e lego (a) go ganetšwa lebollo, (b) go išwa gaMasemola, (c) (Tladi) ke yo bogale, (d) go tšhabela komeng le (e) go hlokofala.

Go ganetšwa lebollo

Tladi o gapeletša gore a lokollwe go yo bolla. Ge a botšwa gore o ganetšwa ke molao wa setšo, o fetola ka go re:

Na koma e fetola matswalo le bogolo bja motho? Aowa, ga e fetole selo. Monyane ke mogolle. Go tla no ba bjalo ka mehla le ge re ka ya mphatong gotee (letl.46).

Gona mo letlakaleng leo, o tšwela pele go nyatša le go nyefola setšo ka gore o re:

Ke molao wa go fahlwa, wa go leka go thibela motho go ba monna mola o sa kgone go mo thibela go gola! Ke molao wa go senya sebaka fela. Le gona mphato wona wo o lebane nna, e sego Monyane.

Ka polelo ye mongwadi o gatelela fela gore go na le dikgopolo tše di ganetšanago mo setsopolweng tše di amago molao, e lego:

- Molao o thibela motho go ba monna.

- Molao ga o kgone go mo thibela go gola.

Ge go le bjalo ke molao wa mohuta mang? Ke ka lebaka leo Tladi bjale a fetolago mawa, a kgopelago gore a išwe komeng gaMasemola. 
Bothata bja ditaba bo thoma gona moo komeng gaMasemola. Kgopolo ye e tiišwa ke mantšu a Dikgati ge a re:

Koma ya kgole ditšhabeng ke dijabatho. Ge re ka leka go go arošetša dileteng, re tla be re go beša gomme o tla swa ra tloga ra ba bomang? (letl.45).

Mohlehle o tiišetša mantšu a Dikgati ka seema ge a re:

Ngwanamaganagobotšwa o wetše komeng tša batho, a re dikoma ke tšešo (letl.44).

\section{Tladi ke yo bogale}

Tladi ke yo bogale ka kudu. Mo go gopolwa ka bašemane ge ba le merakeng. Gona mo Tladi o bolaya Tau a nnoši. Gape go bolelwa ka basetsana bao ba tšwago nokeng, gomme ba opela ka melodi ye mebosana; gomme ba godiša leina la Tladi ka dikoša, ge ba re:

'Na ga re ba rate,

Bosehlwakamafuri,

'Na ga re ba rate,

Bomašiyagodiša.

Re rata mang?

Mogale'a kgomo, (letl.34).

Ka košana ye, Phatudi o phagamišetša maemo a Tladi godimodimo ka go diriša dikwero. O re bašemane ba bangwe ke bosehlwakamafuri, ke bomašiagodiša. Tladi yena ke mogale. Ka lebaka leo go ka thwe maemo a Tladi a phagamišwa ka tirišo ya phapantšho (e lego go fapantšhwa dikgopolo tše pedi. Dikgopolo tše di napile di bolela se sengwe go mmadi (Cuddon, 1998: 191)), ka gore go bolelwa ka dihlopha tše pedi tša bašemane, tšeo di ganetšanago - bofšega le bogale. Ke ka lebaka leo padi ye e ka bitšwago ya bobadi bja phatose. 


\section{Go tšhabela komeng}

Ka lebaka la bonganga, Tladi o gapeleditše, o bile o gorogile morotong, gaMasemola. Bjale go phethagala dikeletšo tša bakgekolo le bakgalabje tša go re bjale koma ya naga dišele e mo jele. Se se tiišetšwa ke leeto la Dikgati ge a yo hlola Tladi gona moo mphatong:

A hwetša Tladi le ba bangwe ba le balwetši kudu. Ba palelwa ruriruri gomme ba šetše ba šala morotong ge koma e eya go fula (letl.53).

Tiragalo ye e tloga e garola matswalo. Ke ya maikutlo a letšhogo a go tliša poifo le mororomelo.

\section{Go hlokofala}

Ditaba di befelela pele ge seemo sa Tladi se gakala ka tsela ya go hlalošwa gore go thwe:

Tladi a golelwa ke bolwetši ka kudu. Matwetwe a lemoga gore molwetši o dikologwa ke phiri gomme a be a lemoga gape gore Dikgati o tsenwe ke maseme (letl.55).

Yona nakong yeo Tladi o ithobalela boroko bjo bogolo - o a hwa. Go hlaloša tiragalo ye, mongwadi o diriša kgakanego bjalo ka kokwane ye bohlokwa ya sengwalo sa phatose. Ke yona e godišago moko wa ditaba sengwalong sa phatose. Kgakanego yeo e tšweletšwa ka tsela ye mo go sehloa sa kanegelo ye:

Jo-nna-jo! Dikgati a hlaba mokgoši gobane o bone ka mahlo ... Banna ba ile ge ba ekwa sello sa Dikgati ba thoma ka pela go hlaba mašata le go opela go tima le go thibela gore sello sa Dikgati se se kwale ka ge e le mokgwa wa komeng go dira bjalo (letl.55). 


\section{Thumo}

Nyakišišo ya taodišwana ye e tsinketše kanegelo ge e nepiša mohuta wa sengwalo sa kwešišano sa go lebana le phatose. Ditiragalo tša phatose di hlalošitšwe ge di lebane le kwelobohloko le manyami a magolo a go nyalana le lehu la molwantšhwa yoo ba lapa le meloko gammogo le karolo ya setšhaba ba ikgwarantšego le yena. Moanegwa yoo o welwa ke ditlaišego le matshwenyego a magolo ka kudu go feta moanegwagolo wa masetlapelo.

Thalošo ya ditiragalo tša sengwalo se sa phatose, e kgonthišitšwe ka go sekaseka kanegelo ya Phatudi ya go ngwalwa ka ngwaga wa 1958 ya go bitšwa Tladi wa Dikgati gore go tle go lemogwe bophelo bja kgakanego le tlabego go moanegwathwadi wa sengwalo sa mohuta wo. Ka lebaka la gore ditiragalo tša phatose di fela di hlakahlakanywa bobading le bosekaseking bja masetlapelo, go bile bohlokwa go fa thalošo ye e tseneletšego ya ditiragalo tša mohuta wo wa sengwalo sa phatose, ka go sekaseka kanegelo yeo ya Phatudi gore go tle go be le lesedi tharollong ya kgakanego le tlabego tša go lebana le bophelo bja kwelobohloko le manyami a magolo dingwalong tša mohuta wo wa phatose.

Ge semelo sa Tladi wa Dikgati, bjalo ka molwantšhwa wa Tladi wa Dikgati, se sekasekwa bjalo ka sengwalo sa kanegelo ya phatose, seo se laolwago ke moko wa ditaba, se lebantšwe le morero woo o hlalošago gore moanegwa yo ke molwantšhwa wa phatose, yoo e lego:

- Motho wa maemo a fase; ke go re motho wa mehleng.

- Motho yoo ga a na bofokodi; ke moloki wa go hloka bošaedi.

- Motho yoo le ge a se na bofokodi o a hlokofala ka lebaka la kgakanego le tlabego.

- Motho yoo o kwelwa bohloko ge a hlokofala ka gore ke motho wa go loka.

Nyakišišo e lemogile gore ditiragalo tša sengwalo sa phatose (ge di bapetšwa le tšeo tša masetlapelo) di lebanywa le go hlokofala ga moanegwa wa mehleng (e sego wa maemo a godimo), wa go hloka maemo setšhabeng. Gantši ditiragalo tšeo di ama lapa le meloko ya lona (bjalo ka lapa la Dikgati le bana ba gagwe). Godimo ga moo, 
se bohlokwa ka ditiragalo tšeo ke kokwane ya go loka. Ke go re ditiragalo di theilwe godimo ga toko le bolo (Tladi o lokile ka gore o na le thompho).

Go tiiša gore ditiragalo tšeo tša Tladi wa Dikgati, di theilwe godimo ga kokwane ya go tšweletša sengwalo sa phatose, go hlokometšwe ge di na le lefetla la:

- $\quad$ Ditiragalo tša lehu la moanegwa wa mehleng

- $\quad$ Ditiragalo tša go ama lapa le meloko

- $\quad$ Ditiragalo bjalo ka kokwane ya go loka

- Ditiragalo tša go hloka bofokodi ebile di tsošago maikutlo a kwelobohloko

- Ditiragalo tša go makatša tša go tšweletša dinnete tša bophelo

- $\quad$ Ditiragalo tša go šiiša namana tša mmele ebile tša go tsoša kwelobohloko ye kgolo

\section{Methopo}

Abrams MH. 1988. A glossary of literary terms. New York: Rinehart \& Winston.

Bal M. 1980. Narratology: Introduction to theory of narrative. Toronto: University of Toronto.

Baldick C. 1990. Oxford dictionary of literary terms. Oxford. New York: Oxford University Press.

Cuddon JA. 1998. A dictionary of literary terms and literary theory. UK. USA: Blackwell Publishers Inc.

Delius P. 1983. The land belongs to us. Braamfontein: Ravan Press (PTY) Ltd.

Draper RD. 1980. Tragedy developments in criticism. London: MacMillan Press Ltd.

Groenewald PS. 1993. Thutadingwalo ya Sesotho sa Leboa 2. Pretoria: Via Afrika.

Holman CH. 1972. A handbook to literature. New York: Odyssey Press.

Jafta DN. 1996. Tragic expression in selected Xhosa literary works. Doctoral thesis.

Stellenbosch, University of Stellenbosch.

Kerkhoff EL. 1962. Kleine deutsche Stilistik. Bern: Franke Verlag.

Lazarus A, Smith HW. 1983. A glossary of literature and composition. Illinois: Urban University Press.

Leech C. 1969. Tragedy. London and New York: Methuen.

Mampuru DM. 1991. Senakangwedi 5. Pretoria: Out of Africa Publishers. 
Marggraff MM. 1994. The moral story in Zulu (1930-1955). MA Dissertation. Pretoria, University of Pretoria.

Mojalefa DD.1994. Tshekatsheko ya hlwayang tsebe. Thesese ya MA. Pretoria, Yunibesithi ya Pretoria.

Mojalefa MJ. 1993. Tshekatsheko ya Sebilwane bjalo ka thetokanegelo, Thesese ya M.A. Pretoria: Yunibesithi ya Pretoria.

Mojalefa MJ. 1995. Pego ye e beakantšhitšwego ya Nyakišišo ya Makxotho (Lekgothwane). Thesese ya Bongaka. Pretoria, Yunibesithi ya Pretoria.

Mojalefa MJ. 1997. Ntthahle ya Boraro (B.A.) Sepedi 302. Pretoria: Yunibesithi ya Pretoria.

Muecke DC. 1982. Irony and the ironic. London: Methuen.

Murfin R, Ray SM. 2009. The Bedford glossary of critical and literary terms. Boston. New York: Bedford/St. Martins.

Oele M. 2007. Aristotle on pathos. Chicago: Loyala University.

Phatudi CN. 1964. Tladi wa Dikgati. Johannesburg: Voortrekker Pers.

Potloane LV. 1994. Pathos and irony in selected Plays by J.M. Synge and Athol Fugard. MA Dissertation. Potchefstroom, Potchefstroom University.

Solivan S. 1998. The spirit, pathos and liberation. Sheffield: Sheffield University Press.

Steiner G. 1961. The death of tragedy. New York: Alfred A Knopf. 


\section{ENGLISH SUMMARY}

\section{SUMMARY}

\section{'Tladi wa Dikgati': A narrative of pathos}

To help resolve confusion arising in analyses of pathos, this article analysed pathos as a moral narrative. The main theme of a narrative of pathos is an intense tragedy that induces pity and sympathy from society, readers and members of the audience (Holman, 1972:384). Narratives of pathos are characterised by the pity and fear aroused by the death of the protagonist, who is regarded by society as ordinary and good. The main character receives pity, more than the main character of a tragedy does.

The events of a narrative of pathos are examined in an analysis of 'Tladi wa Dikgati' (1958) by Phatudi. The narrative presents Tladi as the main character. The following elements of the novella were looked into in the analysis: (a) summary of the novella, (b) the main character, (c) events, (d) elements of pathos, (e) theme, (f) plot analysis, (g) technique, and (h) conclusion. The protagonist's character is analysed from the beginning to the end of the novella in line with the theme of the novel, as one element of pathos. The main character in a narrative of pathos is characterised as an ordinary/common person who is basically good and decent, but who has flaws and makes mistakes that lead to his/her death. His/her death arouses pity and sympathy in society. The events in a narrative of pathos result in the death of an ordinary person, as compared to a tragic narrative where the main character entirely fictional or based on a real-life person. In most cases, the whole family and relatives are affected by the situation. This also happens in 'Tladi wa Dikgati'.

The following factors qualify 'Tladi wa Dikgati' as a narrative of pathos: actions that cause the death of an ordinary person, actions resulting in emotional crisis to family and relatives, actions that show goodwill, ordinary actions that arouse pity and sympathy, actions that portray the realities of life/truths, and tragic actions that arouse emotions of fear and sympathy.

Thus the departure point of the article focuses on Aristotle as the father of tragic narratives. The tragedies Aristotle refers to are quite dissimilar. The article focuses on solutions to problems experienced in distinguishing between tragedy and 
pathos, in African languages. Sepedi literature proves that there are two different types of tragedies, being pathos and tragedy; and pathos being the main focus of this article.

There is a shortage of pathos narratives in Sepedi. The focus of this study is therefore the Sepedi narratives of pathos, which have not previously been investigated in depth. 